\title{
Huge Abscess due to Mycobacterium Abscessus Infection after Breast Augmentation
}

\author{
SeungHwan Hwang ${ }^{1}$, Sujin Bahk ${ }^{1}$, \\ Jae-Woo Chung ${ }^{2}$, Ki Yong Hong ${ }^{1}$, \\ SooA Lim ${ }^{1}$, SuRak Eo ${ }^{1}$ \\ ${ }^{1}$ Department of Plastic and \\ Reconstructive Surgery, Dongguk \\ University Ilsan Hospital, Dongguk \\ University Graduate School of Medicine, \\ Goyang; ${ }^{2}$ Department of Laboratory \\ Medicine, Dongguk University Ilsan \\ Hospital, Dongguk University Graduate \\ School of Medicine, Goyang, Korea
}

Periprosthetic capsular contracture, implant rupture, and deflation are well-known delayed complications of augmentation mammaplasty. However, infection remains the most common cause of reoperation after breast implant surgery. We report the case of a nontuberculous mycobacterial infection with huge abscess formation after augmentation mammaplasty. A 29-year-old woman visited our clinic with enlarged breasts after undergoing breast augmentation at a local clinic 4 years ago. She had no pain and tenderness, except some hardness around the breast margin. Nine months after surgery, her breasts began to grow larger, but showed no other typical symptoms, which led the patient to neglect the enlargement. After exploring through an inframammary approach, a large amount of serous fluid leaked out on both sides and we identified a huge abscess in a pocket localized in the submuscular plane. During exploration, the infected implants, which contained a pus-like fluid with a foul odor, were completely drained. The specimen culture revealed growth of Mycobacterium abscessus, which is a rare cause of infections after breast augmentation. While the overall incidence of mycobacterial infections after breast augmentation is low, our case demonstrates that huge abscess and granuloma formation should be considered as a potential complication of breast surgery.

Keywords Abscess, Breast Implantation, Mycobacterium, Nontuberculous mycobacteria
No potential conflict of interest relevant to this article was reported. surgical exploration is required in $3 \%$ of all breast implant patients $[5,6]$. Infections are typically caused by bacterial skin flora, specifically Staphylococcus epidermidis, S. aureus, and coagulase-negative staphylococci [7-9]. In analyses of risk factors for recurrent implant infections, various researchers have reported that infections of $S$. aureus were more likely to cause device failure than infections with other bacteria [10]. Breast infections caused by mycobacteria after breast augmentation are not common, but are increasingly reported. However, few reports have been published of nontuberculous mycobacterial (NTM) infections associated with breast implants. Herein, we report an NTM infection with huge abscess formation after augmentation mammaplasty.

\section{CASE REPORT}

A 29-year-old woman visited our clinic with enlarged breasts after undergoing bilateral breast augmentation at a local clinic 4 years ago (Fig. 1). She had no pain and tenderness, except some hardness around the breast margin, and fluctuation in size was noted on both
Received: Jun 14, 2018 Revised: Jun 27, 2018 Accepted: Jul 4, 2018

Correspondence: SuRak Eo Department of Plastic and Reconstructive Goyang 10326, Korea. E-mail: sreodoc@gmail.com

Copyright (C) 2018 The Korean Society for Aesthetic Plastic Surgery.

This is an Open Access article distributed under the terms of the Creative Commons At tribution Non-Commercial License (http://creativecommons.org/licenses/by-nc/4.0/) which permits unrestricted non-commercial use, distribution, and reproduction in any medium, provided the original work is properly cited. www.e-aaps.org 
sides. She had regular menstrual periods and had no previous breast disease or medical history. Three months after surgery, a clinical examination revealed that palpable and deforming periprosthetic capsular contracture had developed. There was no redness, induration, or swelling in either breast. After 6 months, her breasts began
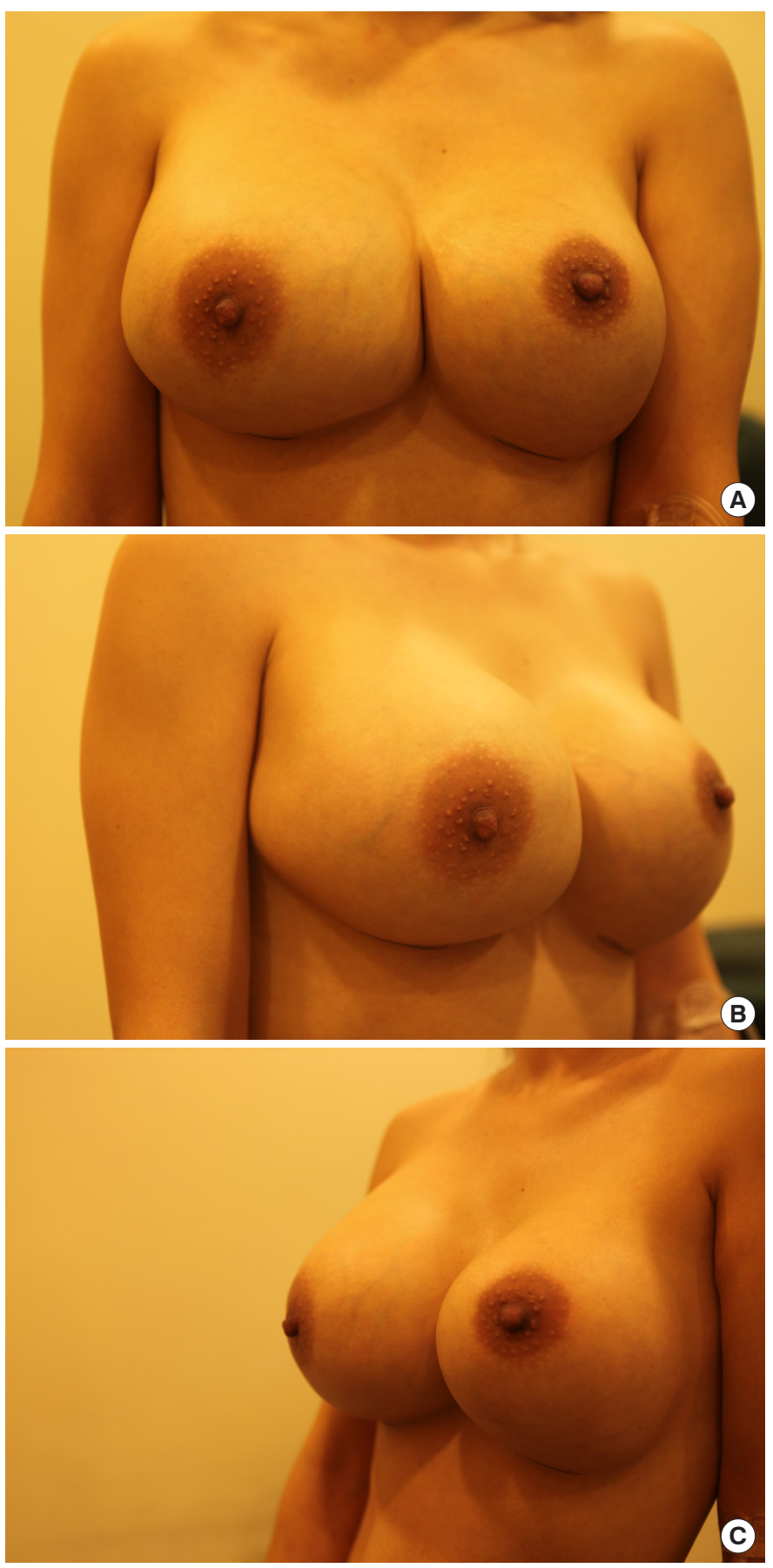

Fig. 1. External views of enlarged breasts before exploration. A 29year-old woman visited our clinic with enlarged breasts after breast augmentation at a local clinic 4 years ago. (A) Anteroposterior view. (B) Left oblique view. (C) Right oblique view. to grow larger, but showed no other typical symptoms, which led the patient to neglect the enlargement. Consequently, since she had not experienced any improvement of her symptoms, she chose to undergo removal of her breast implants via capsulectomy. After exploring through an inframammary approach, a large amount of serous fluid leaked out on both sides, and we identified a huge abscess in a pocket localized in the submuscular plane. The infected implants, which contained a pus-like fluid with a foul odor, were completely removed (Fig. 2). A pathologic examination to evaluate the possibility of anaplastic large-cell lymphoma (ALCL) through seroma immunohistochemical staining showed negative results. Her abscess culture was sterile upon routine bacterial culturing, which led us to consider the possibility of an atypical infection, such as a mycobacterial infection. Fluid subcultures were performed using sheep blood agar, and the plates were incubated at $35^{\circ} \mathrm{C}$ for 48 hours. After incubation, sheep blood agar yielded non-hemolytic,
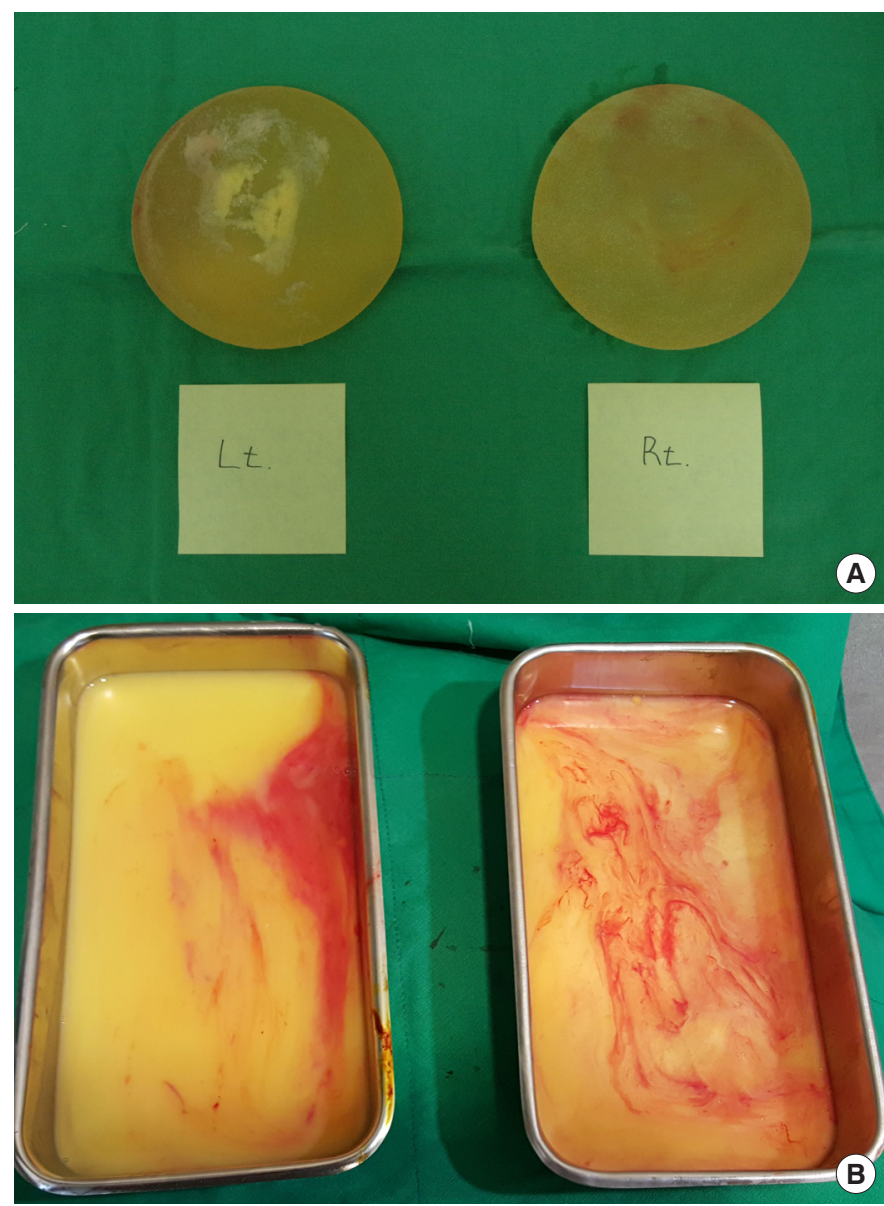

Fig. 2. Infected implants and pus-like fluid. During exploration, the infected implants, which contained a pus-like fluid with a foul odor, were completely drained. (A) Allergan textured round implants were removed during exploration. (B) Pus-like serous fluid was drained from both pockets localized in the submuscular plane. Lt., left; Rt., right. 


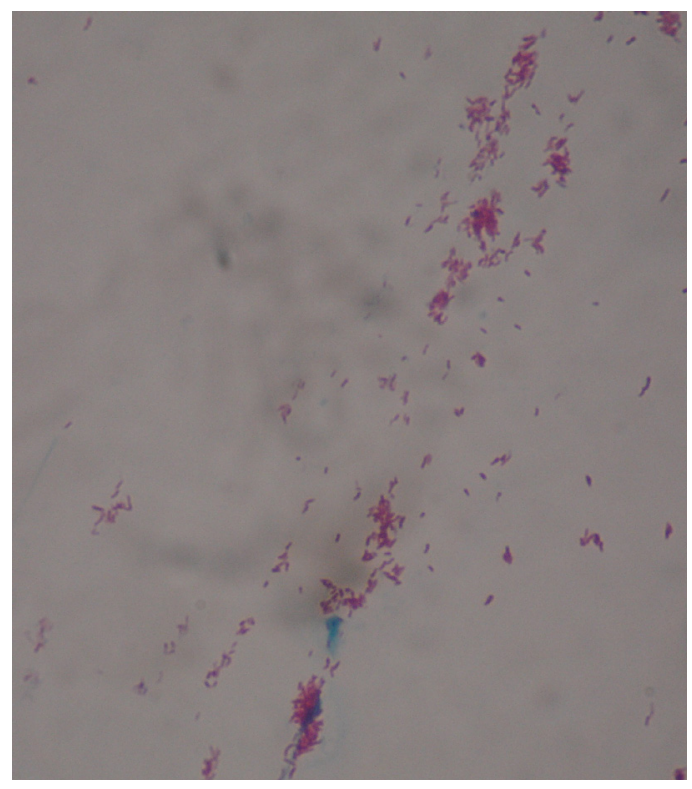

Fig. 3. Microscopic appearance of the isolate (acid-fast stain, $\times 1,000$ ). Sheep blood agar yielded non-hemolytic, white pinpoint colonies, and we performed acid-fast bacilli staining.

white pinpoint colonies and we performed acid-fast bacilli staining (Fig. 3). Polymerase chain reaction and sequencing for the $16 \mathrm{~S}$ rRNA gene was performed by Macrogen (Seoul, Korea) using a $16 \mathrm{~S}$ rRNA $785 \mathrm{~F}$ and $907 \mathrm{R}$ primers. The corresponding 1,449-bp contig was compared to the sequencing results using the basic local alignment search tool with the EzTaxon database [11], and identified as $M y$ cobacterium abscessus, a rare cause of infections after breast augmentation. For financial reasons, the patient did not want to undergo additional evaluations, such as computed tomography or magnetic resonance imaging.

After 4 weeks of antibiotic therapy, her breasts showed clinical improvements. The patient had not experienced any further recurrence of the infection at a 1-year follow-up.

\section{DISCUSSION}

Breast implants are widely used for cosmetic and reconstructive purposes $[1,2,11]$. However, reoperation often occurs after implantation, most often due to infection. Periprosthetic infection is the most feared complication of implant procedures [12]. Pathogens such as mycobacteria, coagulase-negative staphylococci, and S. aureus are the most common cause of surgical site infections [12-14]. However, this case emphasizes the possibility of identifying $M$. $a b$ scessus as an atypical pathogen involved in infections after breast implantation.

The M. abscessus complex comprises a group of rapidly growing, multidrug-resistant, nontuberculous mycobacteria that are responsible for a wide spectrum of skin and soft tissue diseases, central nervous system infections, bacteremia, and ocular and other infections [15]. Much more research is required regarding infection control for breast implants, but it is generally accepted that early removal is necessary to prevent tissue necrosis and contracture [16].

While the overall incidence of mycobacterial infections after breast augmentation is low, our case demonstrates that huge abscess and granuloma formation should be considered as a potential complication after breast implant surgery. Successful treatment was achieved through an early and aggressive surgical intervention. The accurate diagnosis of a mycobacterial infection requires the organism to be identified in a sample of the periprosthetic tissue. Therefore, if a mycobacterial infection is suspected, specimens must be submitted for acid-fast staining and mycobacterial culture, in addition to standard bacterial analyses [12]. In this case, the possibility of an atypical infection, such as a mycobacterial process, was considered because multiple specimens of fluid from both breasts, debridement tissue, and prosthesis were aseptic in routine bacterial and fungal cultures. Subsequent specimens from the ablated prosthesis underwent mycobacterial culture testing, and M. abscessus was identified as the causative pathogen. Therefore, we administered long-term antimycobacterial therapy to eradicate this atypical infection.

Breast implants are associated with an increased risk of breast ALCL. If a patient has late-onset periprosthetic seroma, a pathologic examination for ALCL should be performed. The assessment for ALCL should include a cytological evaluation of seroma with Wright Giemsa-stained smears and cell block immunohistochemical testing for cluster of differentiation and anaplastic lymphoma kinase markers [17].

\section{CONCLUSION}

In conclusion, the main contribution of this report is a description of the diagnosis and successful removal of breast prostheses with an atypical NTM infection after breast augmentation. Surgical removal and antibiotic treatment were successful, as shown by the patient's subsequent uneventful course. Our case underscores that a high level of suspicion of rare pathogens such as atypical mycobacteria is needed when considering periprosthetic infections that do not improve despite prolonged treatment.

\section{PATIENT CONSENT}

The patient provided written consent for the use of her images.

\section{REFERENCES}

1. Cohen JB, Carroll C, Tenenbaum MM, et al. Breast implant-associated infections: the role of the national surgical quality improvement program and the local microbiome. Plast Reconstr Surg 2015;136:921-9. 
2. American Society of Plastic Surgeons. 2013 plastic surgery statistics report [Internet]. Arlington Heights, IL: American Society of Plastic Surgeons; c2013 [cited 2014 Aug 23]. Available from https://www. plasticsurgery.org/documents/News/Statistics/2013/plastic-surgerystatistics-full-report-2013.pdf.

3. Feldman EM, Kontoyiannis DP, Sharabi SE, et al. Breast implant infections: is cefazolin enough? Plast Reconstr Surg 2010;126:779-85.

4. Persichetti P, Langella M, Marangi GF, et al. Periprosthetic breast abscess caused by Streptococcus pyogenes after scarlet fever. Ann Plast Surg 2008;60:21-3.

5. Phillips BT, Bishawi M, Dagum AB, et al. A systematic review of antibiotic use and infection in breast reconstruction: what is the evidence? Plast Reconstr Surg 2013;131:1-13.

6. Reish RG, Damjanovic B, Austen WG Jr, et al. Infection following implant-based reconstruction in 1952 consecutive breast reconstructions: salvage rates and predictors of success. Plast Reconstr Surg 2013; 131:1223-30.

7. Ahn CY, Ko CY, Wagar EA, et al. Microbial evaluation: 139 implants removed from symptomatic patients. Plast Reconstr Surg 1996;98:1225-9.

8. Dobke MK, Svahn JK, Vastine VL, et al. Characterization of microbial presence at the surface of silicone mammary implants. Ann Plast Surg 1995;34:563-9.

9. Adams WP Jr, Conner WC, Barton FE Jr, et al. Optimizing breast pocket irrigation: an in vitro study and clinical implications. Plast Reconstr Surg 2000;105:334-8; discussion 9-43.

10. Spear SL, Seruya M. Management of the infected or exposed breast prosthesis: a single surgeon's 15-year experience with 69 patients. Plast Reconstr Surg 2010;125:1074-84.

11. Yoon SH, Ha SM, Kwon S, et al. Introducing EzBioCloud: a taxonomically united database of $16 S$ rRNA gene sequences and whole-genome assemblies. Int J Syst Evol Microbiol 2017;67:1613-7.

12. Vinh DC, Rendina A, Turner R, et al. Breast implant infection with Mycobacterium fortuitum group: report of case and review. J Infect 2006;52:e63-7.

13. Betal D, Macneill FA. Chronic breast abscess due to Mycobacterium fortuitum: a case report. J Med Case Rep 2011;5:188.

14. Olsen MA, Lefta M, Dietz JR, et al. Risk factors for surgical site infection after major breast operation. J Am Coll Surg 2008;207:326-35.

15. Lee MR, Sheng WH, Hung CC, et al. Mycobacterium abscessus complex infections in humans. Emerg Infect Dis 2015;21:1638-46.

16. Haiavy J, Tobin H. Mycobacterium fortuitum infection in prosthetic breast implants. Plast Reconstr Surg 2002;109:2124-8.

17. de Boer M, van Leeuwen FE, Hauptmann M, et al. Breast implants and the risk of anaplastic large-cell lymphoma in the breast. JAMA Oncol 2018;4:335-41. 